\title{
Adaptively Reevaluated Bayesian Localization (ARBL): A Novel Technique for
} Radiological Source Localization

\author{
Erin A. Miller ${ }^{(\mathrm{a})}$, Sean M. Robinson ${ }^{(\mathrm{b})}$, Kevin K. Anderson ${ }^{(\mathrm{a})}$, Jonathon D. McCall ${ }^{(\mathrm{a})}$, Amanda M.
} Prinke $^{(a)}$, Jennifer B. Webster ${ }^{(a)}$, Carolyn E. Seifert ${ }^{(a)}$

(a) Pacific Northwest National Laboratory, Richland, Washington, 99352, United States

(b) Pacific Northwest National Laboratory, Seattle, Washington, 98109, United States

\section{Abstract}

We present a novel technique for the localization of radiological sources in urban or rural environments from an aerial platform. The technique is based on a Bayesian approach to localization, in which measured count rates in a time series are compared with predicted count rates from a series of pre-calculated test sources to define likelihood. This technique is expanded by using a localized treatment with a limited Field of View (FOV), coupled with a likelihood ratio reevaluation, allowing for real-time computation on commodity hardware for arbitrarily complex detector models and terrain. In particular, detectors with inherent asymmetry of response (such as those employing internal collimation or self-shielding for enhanced directional awareness) are leveraged by this approach to provide improved localization. Results from the localization technique are shown for simulated flight data using monolithic as well as directionally-aware detector models, and the capability of the methodology to locate radioisotopes is estimated for several test cases. This localization technique is shown to facilitate urban search by allowing quick and adaptive estimates of source location, in many cases from a single flyover near a source. In particular, this method represents a significant advancement from earlier methods like full-field Bayesian likelihood, which is not generally fast enough to allow 
1 for broad-field search in real time, and highest-net-counts estimation, which has a localization

2 error that depends strongly on flight path and cannot generally operate without exhaustive

3 search.

4 Keywords: aerial search, radiological search, gamma detection, localization, maximum

5 likelihood, Bayesian

\section{$6 \quad 1.0$ Introduction}

7 Aerial search is an important tool to locate lost or missing radiological sources and map

8 radiation. Aerial surveys using helicopter-based detection systems represent one approach to

9 radiological search that can allow for rapid results in an urban environment. However, this

10 approach has many challenges inherent to the use of a high-standoff detector at high speed.

11 Rapidly-varying background in a complex urban environment, the presence of confounding

12 NORM objects, and medical sources all present significant potential for confusion. Additionally,

13 the field of view from an airborne detector will cover a large area, allowing for many of these

14 confounding sources to simultaneously affect measurement [1-4].

15 At present, many algorithms exist to perform source detection or identification based on spectral

16 ID or anomaly methods [5, 6]. However, efforts at source localization for airborne detection are

17 limited to inferences based on the location of maximum count rate. Because of this, locating

18 sources requires an exhaustive search using many airborne passes, or secondary (i.e. ground-

19 based) inspection, both of which are time-consuming. Thus there is a need to develop numerical

20 techniques for airborne localization which are applicable to a wide variety of environments,

21 including dense and complex urban areas. These techniques are expected to require a good

22 estimate of background, a detector with wide-area search capability and directional sensitivity, 
1 and an estimate of detector response to sources in the environment, such that the observed data

2 can be correlated with detector response to generate an estimate of source location.

\section{$3 \quad 2.0$ Methods}

4 We seek to develop a method which will localize a source in real-time or near-real-time using an

5 arbitrarily complex directional detector and incorporating what may be a very complex local

6 topography with correspondingly complex background radiation.

$7 \quad 2.1$ Flight Scenarios and Hardware

8 Aerial survey from a helicopter typically takes place at an altitude of 50-200 m, which may vary

9 during any given flight. Conventionally the field of view is approximately 45 degrees, as shown

10 in Fig. 1, although this can vary with detector design or for very strong sources. For max-counts-

11 based localization, this sets the approximate spatial accuracy. Helicopter velocity is variable as

12 well but typically ranges between 50 and $80 \mathrm{kts}$.

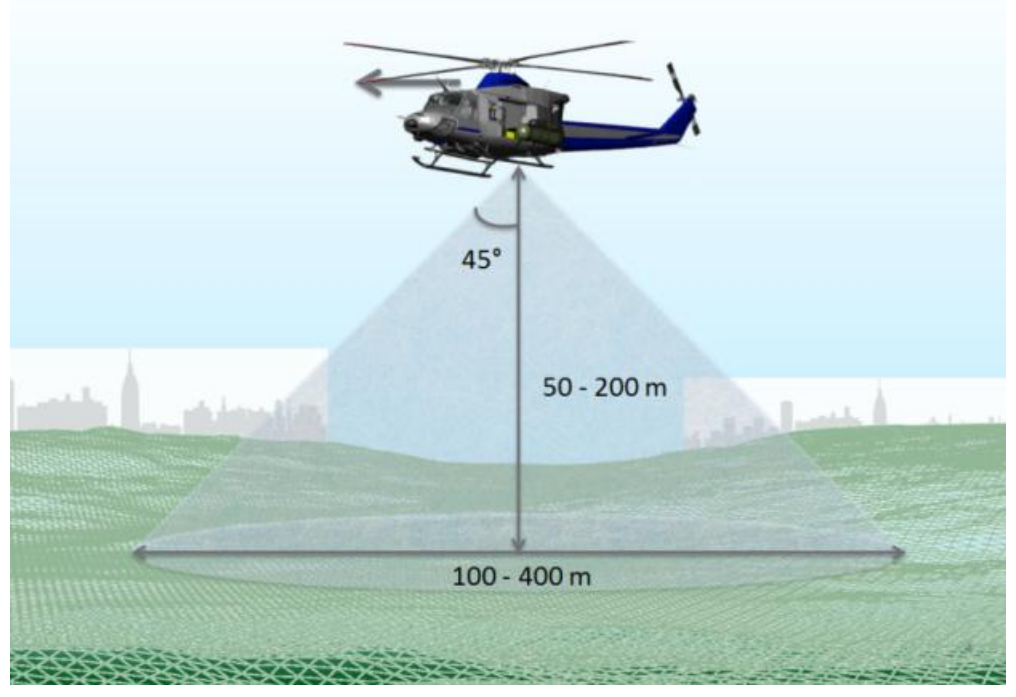

14 Figure 1. Typical flight parameters. 
2 The localization approach discussed here can be applied to a wide range of detector designs.

3 Here, we focus on the Leidos HeliSORDS detector, an array of 92 CsI crystals designed to

4 provide directional information or imaging while maintaining a high count rate (Fig 2.). The

5 crystals are arranged into two pods on either side of the helicopter, with 2 assemblies in each

6 pod. A single assembly consists of 23 crystals arrayed in an inner semicircle and an outer

7 semicircle; each crystal in the inner semicircle is positioned to be illuminated directly by a

8 source at some angles but occluded by the outer crystals at other angles. This produces a

9 directionally dependent signature: the response of each inner crystal shows a peak at a different

10 roll angle, approximately $60^{\circ}$ wide, with two smaller sidelobes. Additional directional

11 information is provided by occlusion of the outer crystals by each other, by preferential

12 occlusion of one pod over another by the aircraft (especially at high angles), and Compton

13 imaging based on processing coincidence events between multiple crystals. For simplicity, we

14 do not include Compton imaging in the analysis presented here, although the approach could be 15 generalized to include it. 


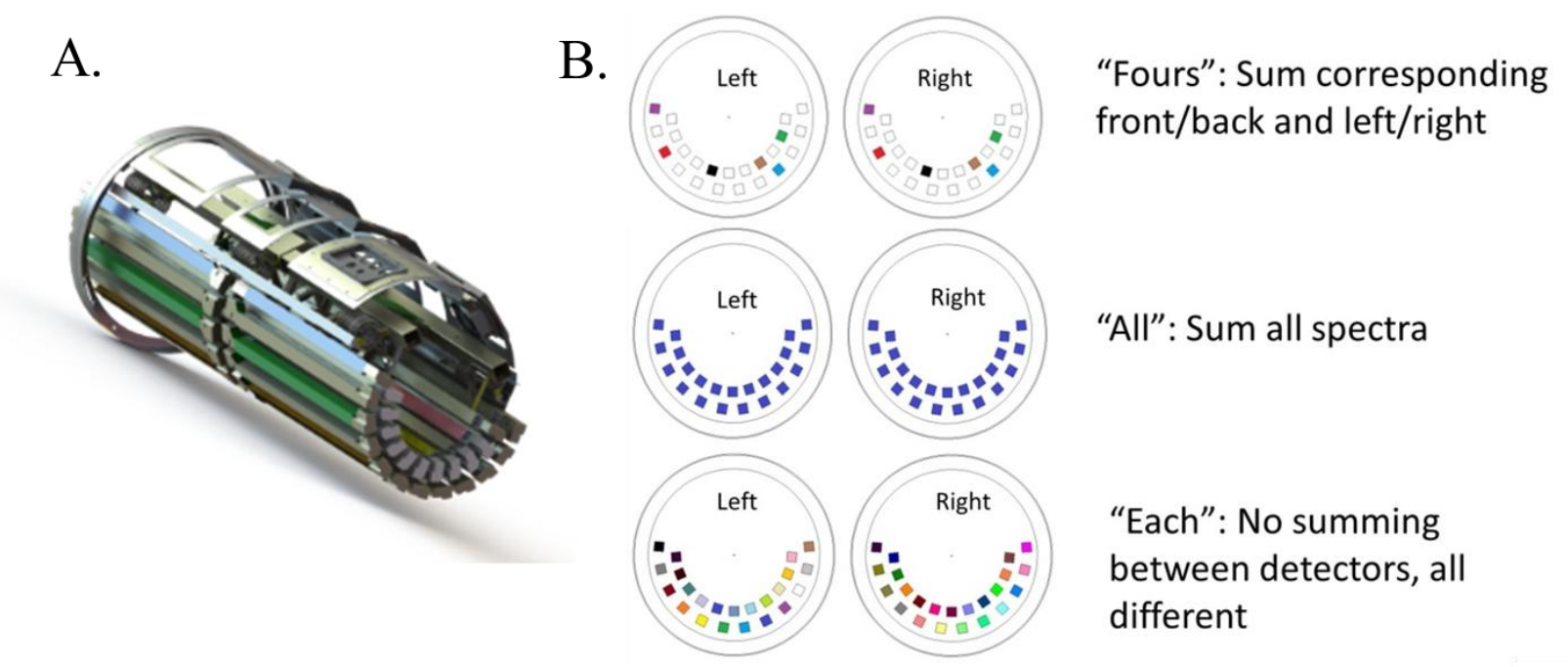

2 Fig. 2. Leidos HeliSORDS detector. A. Arrangement of CsI crystals in a single pod (consisting

3 of a front and back assembly). B. Cross sections of a left and right assembly, indicating binning

4 schemes for combining data across multiple crystals.

5 In order to assess the sources of directional information and to improve efficiency, we consider

6 three different binning schemes for the 92 crystal array, as shown in Figure 2b. At one extreme

7 is the "Each" case, where no binning is done and each crystal is used individually to produce

8 results. This provides the maximum possible directional information (short of including

9 Compton imaging) but may be computationally inefficient. The "Fours" case sums the crystals

10 in groups of four: front left, back left, front right, and back right. This approach is intended to

11 improve computational efficiency of the entire method while only summing the crystals likely to

12 produce similar results, effectively preserving directional sensitivity. This gives a single

13 assembly of 23 effective crystals, but will tend to average out effects such as attenuation or

14 scatter within the aircraft itself. Finally, the "All" case sums all crystals together, removing most

15 directional information. 
2 Estimates of the detector response to sources at any relative location and distance are used for

3 scenario generation and initial algorithm testing. These estimates also provide an input to the

4 maximum likelihood method described below, in which observed data is compared with a set of

5 hypotheses about a source. Because detailed spectral information including downscatter and

6 partial energy deposition in the detectors may be needed, a simple ray-trace or attenuation-only

7 model may not be sufficient for the general case. We construct detailed simulations by

8 separating the radiation transport problem into pieces: 1) interaction of a point source with the

9 ground, or, for background, interaction of a pre-calculated background spectrum with the

10 ground; 2) transport of radiation over large distances to the aircraft; and 3) transport of radiation

11 through the aircraft and in the detector itself. Steps 1 and 2 are performed using discrete

12 ordinates finite element software (Attila, Transpire, Inc.), while detector response is calculated

13 using Monte Carlo methods (MCNP). Transport calculations are done for source-detector pairs

14 encompassing a range of isotopes, altitudes, offsets, and ground normal vectors; plus roll, pitch,

15 and yaw at the detector. This forms a library which can be drawn from both for creating detailed

16 scenarios and for testing expected detector responses.

\section{$17 \quad 2.2$ Maximum Likelihood Localization}

18 To operate on an airborne detection system, the localization algorithm must be possible in (near)

19 real time, and must locate multiple sources of interest over a wide area while filtering out known

20 background sources. To accomplish this, this work employs Maximum-Likelihood based

21 localization techniques. This sort of approach has been employed to look for radioisotope

22 sources in many search applications, which data was been combined from single or networked 
1 radiation detectors to achieve source localization in real time or after the fact [7-11]. Generally,

2 these approaches are based on a formalism where data from each detector or time step is

3 compared with the expected data from every notional source location to determine the most

4 likely location. A general treatment of this sort of Maximum Likelihood Estimation (MLE)

5 approach is shown in Figure 3 for a moving detector in an arbitrary 3-D region which may

6 contain a radiological source. Here, the series of measured values is compared with the

7 computed response to a test source with given location and strength parameters. To make a

8 visual map of results, test source responses can be calculated on a grid of locations for a range of

9 source parameters, to provide a visual tool for localization.

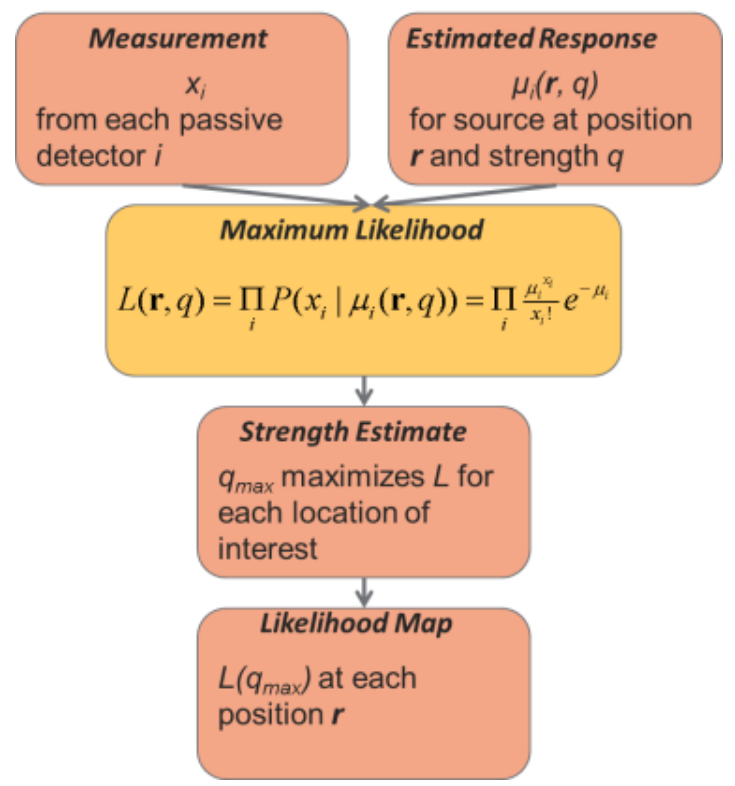

11 Fig. 3. Maximum Likelihood algorithm for a Poisson-distributed variable.

$12 \quad$ 2.2.1 Bayesian updating

13 The maximum likelihood calculation described above can easily be understood in terms of a

14 fixed set of measurements. For aerial search, however, we want a likelihood map which can

15 update continuously as new measurements are added. To do this we consider a Bayesian 
1 approach in which updates to the likelihood map are made as new data points are acquired. An

2 initial prior distribution is assumed, with unit value throughout space and strength. At each time

3 step, a likelihood map is produced corresponding to a single measurement and multiplied by the

4 prior distribution. This allows the likelihood map to evolve as new measurements are included.

$5 \quad$ 2.2.2 Simplified Source Strength Dependence

6 The likelihood formulation above can be used to calculate a probability map in a straightforward

7 fashion by checking test sources as a function of position on the ground and source strength.

8 However, test source response as a function of source strength can be described analytically:

$$
\mu_{i}(\mathbf{r}, q)=b_{i}+q s_{i}(\mathbf{r})
$$

where $b_{i}$ is the background count rate at detector position $i, q$ is the source strength, and $s_{i}(\boldsymbol{r})$ is

11 the count rate at detector position $i$ due to a unit-strength source at location $r$. Substituting this

12 back into the likelihood calculation in Fig. 3, approximating the distribution as Gaussian rather

13 than Poisson (with a variance equal to the count rate from the hypothesized test source), and

14 taking the log, we have

15

$$
\log (L(\mathbf{r}, q)) \approx-\frac{1}{2} \sum_{i} \log \left(2 \pi\left(b_{i}+q s_{i}(\mathbf{r})\right)+\frac{\left(x_{i}-\left(b_{i}+q s_{i}(\mathbf{r})\right)\right)^{2}}{b_{i}+q s_{i}(\mathbf{r})}\right.
$$

17 quadratic, choosing the coefficients for each location and time based on calculations at 3

18 different $q$-values (in practice, $0,7.5$, and $15 \mathrm{mCi}$; although the results are quite insensitive to the 19 choice of sampled values). 
1 Changing the likelihood calculation to use a quadratic approximation results in approximately a

2 factor of 8 decrease in computational time, due to the reduction in calculation from 24 to 3

3 discrete source strengths. This case is a $10 \mathrm{mCi}{ }^{137} \mathrm{Cs}$ source, sampled with $20 \mathrm{~m}$ pixels and a 300

$4 \quad \mathrm{~m}$ field of view. The resulting likelihood calculation is in excellent agreement with the previous

5 version. In most locations, differences between the two results are vanishingly small compared

6 with the variations due to measurement noise. The only exceptions to this were seen in regions

7 where the estimated source strength was very high (usually at the boundaries of a measured

8 region); in this case, the discretely sampled version artificially limited the source strength leading

9 to an incorrect answer, while the quadratic fit did not require a pre-defined strength range.

\subsubsection{Limited Field of View and Likelihood Ratio Test}

11 A single aerial flight may cover a very large area - typically multiple kilometers. However, each

12 individual measurement is expected to be physically relevant over a much smaller field of view,

13 except perhaps in the case of an extremely strong source. Both physically and computationally,

14 it makes sense to limit the portion of the likelihood map which is updated with each new

15 measurement, rather than recomputing a full map which may be several kilometers across.

16 However, this naturally leads to different points on the map being informed by a different

17 number of measurements. This tends to drive down the likelihoods for more heavily-measured

18 regions, since each individual probability is less than 1. This effect can be seen in Fig. 4 A and

$19 \mathrm{~B}$, which show the likelihood in a line along the flight path and lateral to it. The true source

20 location, towards the middle, shows a local maximum, but the endpoints which are less-heavily

21 measured also show high values. As the field of view is varied, the likelihood in any given

22 region changes significantly. 

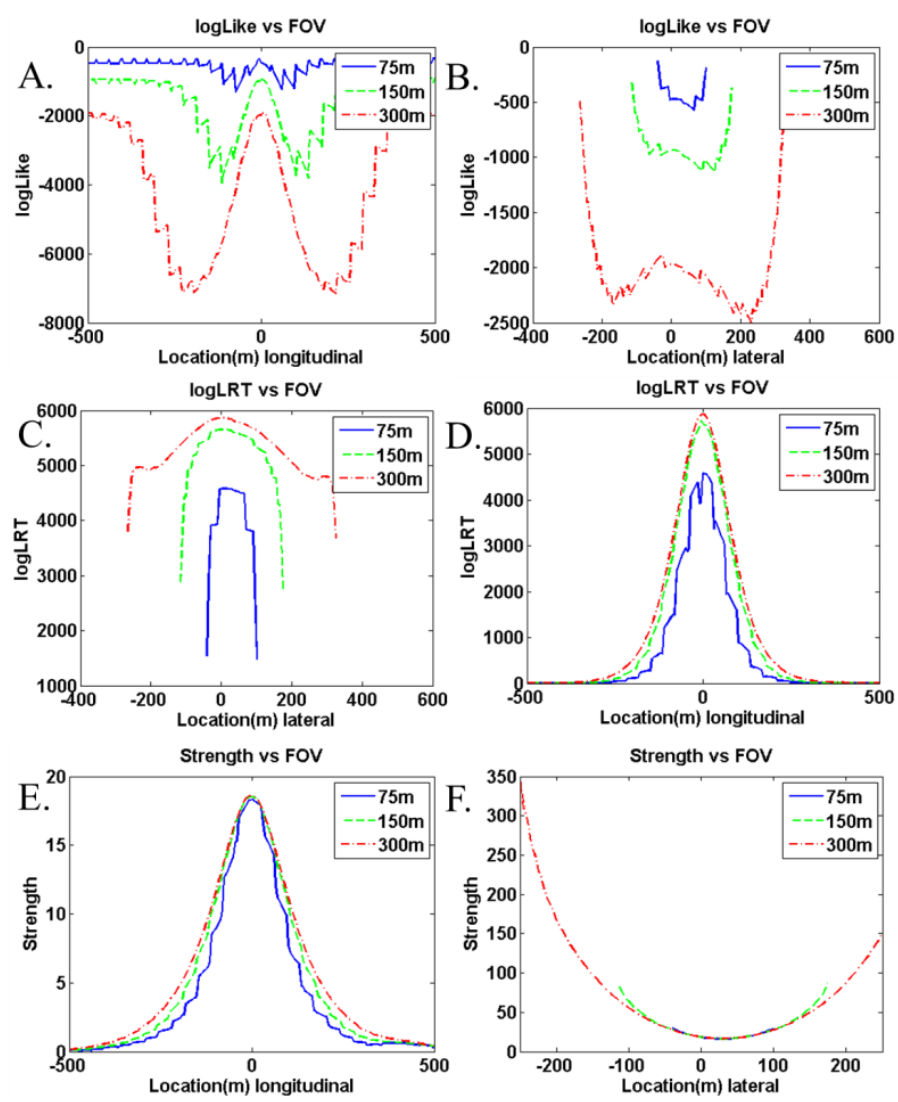

2 Fig. 4. Behavior of log-Likelihood, log-LRT, and strength estimate as a function of field of

3 view. Left column is on a line parallel the flight path passing through the source at $0 \mathrm{~m}$; right

4 column is on a line perpendicular to the source and passing through the source at $0 \mathrm{~m}$.

5 To address this, we calculate the likelihood ratio, comparing the value of likelihood at the most

6 likely source strength to the likelihood of a zero strength source. This produces a measure of

7 confidence in the result; as a normalization, this is effective at countering the cumulative effects

8 of multiple measurements since the value of the likelihood for a zero strength source is the same

9 as for a very distant source, so the metric is insensitive at a given point to very distant measurements. Behavior of the likelihood ratio test as a function of field of view is shown in

11 Fig. $4 \mathrm{C}$ and D. As the field of view is increased, the LRT along the direction of the flight path

12 converges. Convergence also takes place lateral to the flight path, but is slower than along the 
1 path since points at large distance are covered by fewer measurements. Finally, we see that the

2 estimates of source strength which are produced are also consistent as field of view is increased,

3 and converge to a smooth distribution (Figure $4 \mathrm{E}$ and $\mathrm{F}$ ).

4 The importance of a limited field of view to computational time is straightforward, since

5 computational time is largely governed by the number of times the likelihood or likelihood ratio

6 is computed when a new measurement is added. With the quadratic approximation, three

7 strengths are checked per location. The number of locations updated per measurement $N$ is set

8 by

$$
N=\pi(F O V / \text { step size })^{2}
$$

For a $150 \mathrm{~m}$ field of view sampled in $5 \mathrm{~m}$ increments, this means updating just under 3000

11 locations per second, as compared to 33,000 per second if the entire region (in this case, a single

12 pass $2500 \mathrm{~m}$ long) is considered. This also allows the size of the map to "grow" adaptively as

13 new regions are sampled during the flight, rather than requiring that the region to be measured be

14 defined prior to the flight. For a $150 \mathrm{~m}$ field of view with $5 \mathrm{~m}$ pixels and 23 effective detector

15 modules this runs at approximately 5.7 points/sec on a desktop machine (8 core, $3.2 \mathrm{MHz}$ ).

\subsection{Results and Discussion}

17 The enhancements described in the preceding sections are combined in a single approach called

18 the Adaptively Reevaluated Bayesian Localization (ARBL) algorithm. We examine the

19 performance of ARBL using simulated data. This approach allows us to vary the scenario in a

20 controlled fashion and to readily perform ensemble tests with multiple noise realizations. We

21 begin with a simulated dataset for an aircraft at $83 \mathrm{~m}$ altitude travelling at $70 \mathrm{kts}$ in a straight line,

22 with data reported at 1-second intervals. A "flat" radiological background is used consisting of a 
1 fixed overall composition of Potassium, Uranium, and Thorium (KUT) based on realistic

2 background values measured in the Government Wash area in Nevada and varied according to

3 Poisson noise; for a sense of scale, at the measured point nearest the true source location, a 20

$4 \mathrm{mCi}^{137} \mathrm{Cs}$ source increases the count rate by $44 \%$ of the background. The algorithm is designed

5 to be flexible as to the method of background estimation, whether based on a rolling average of

6 prior measurements or a priori knowledge of the area being flown. For these initial tests, the

7 algorithm uses an average of the first few seconds of data to estimate the background. Likewise,

8 ARBL can be implemented by considering each energy in the gamma spectrum independently,

9 by using selected energy bins, or even using a spectral anomaly metric directly to create the

10 likelihood map, but here we restrict our testing to gross counts. A $10 \mathrm{mCi}{ }^{137} \mathrm{Cs}$ source is placed

11 on the ground. ARBL is run using $10 \mathrm{~m}$ pixels and a $125 \mathrm{~m}$ FOV. We examine the logLRT after

12 a single pass, $25 \mathrm{~m}$ offset from the source laterally, and again after a second pass in the opposite

13 direction, $25 \mathrm{~m}$ to the other side of the source. The resulting logLRT maps are shown in Fig. 5.

14 The line plots on the left show the logLRT values on a line passing through the source

15 perpendicular to the flight path direction, while the plots on the right show a 2-D color map for

16 the "fours" binning. 


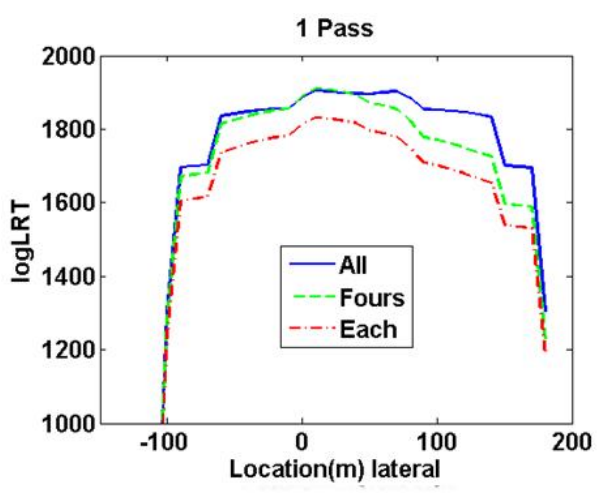

Single Pass, $10 \mathrm{mCi}^{137} \mathrm{Cs}$

"fours" method
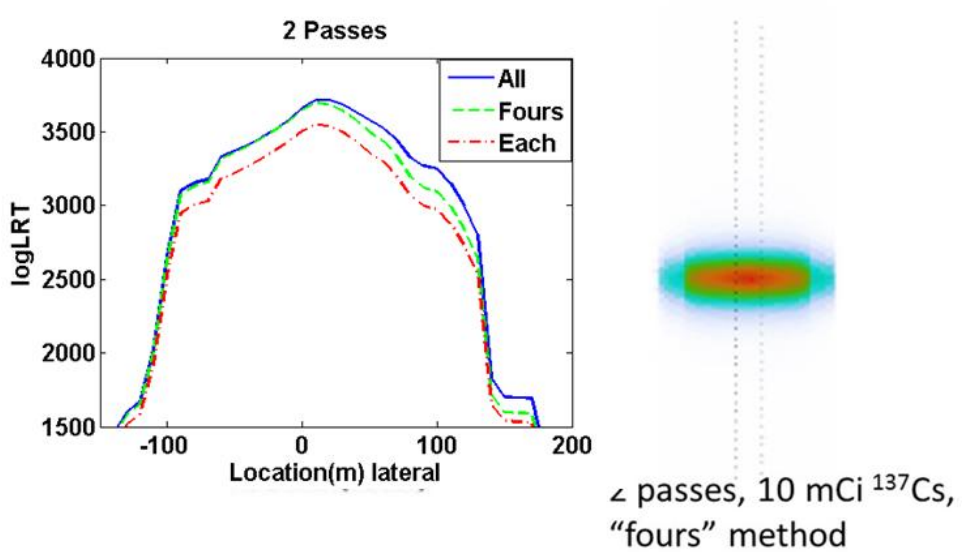

1

2 Fig. 5. ARBL results for one and two passes in the vicinity of a $10 \mathrm{mCi}{ }^{137} \mathrm{Cs}$ source. The line

3 plots on the left show log-likelihood values along a line through the true source location (at $0 \mathrm{~m}$ )

4 and perpendicular to the flight path direction.

5 After a single pass, the likelihood indicates the source position along the flight direction clearly.

6 Lateral to the path the behavior is a bit more complex. If all detector crystals are binned together

7 ("All"), the likelihood shows two peaks on either side of the flight path, at equal distance from

8 the detector with a broad elevated region between. This is characteristic of a non-directional

9 detector, in which the fundamental "symmetry" between the data from a source to the right or the

10 left is unbroken. Using all 92 crystals separately ("Each”) or binning into a single 23-crystal

11 assembly ("Fours") breaks this symmetry and correctly identifies the side of the detector 
1 containing the source. The overall shape of the logLRT is similar in each case, but the

2 confidence is slightly lower for "Each" since it incorporates more and noisier detectors. After

3 two passes on either side of the source, the overall confidence has gone up in all three cases, and

4 the directional information plays a much less important role in locating the source; all three

5 binning schemes show a similar shape and correctly identify the source location.

6 In order to understand localization accuracy we study an ensemble of datasets with the same

7 basic setup but different statistical realizations, simulated by repeatedly injecting source

8 signatures plus poisson noise into measured background. The best estimated source locations for

9 an ensemble of 10 realizations of a single aircraft passs, offset $50 \mathrm{~m}$ from the true source, are

10 shown in Figure 6. The true source is shown as a black plus. The blue plusses indicate the

11 maximum gross counts locations, and also serve to illustrate the aircraft location. ARBL results

12 are shown in red, with a plus indicating "All", a circle indicating "Fours", and an asterisk

13 indicating "Each". 

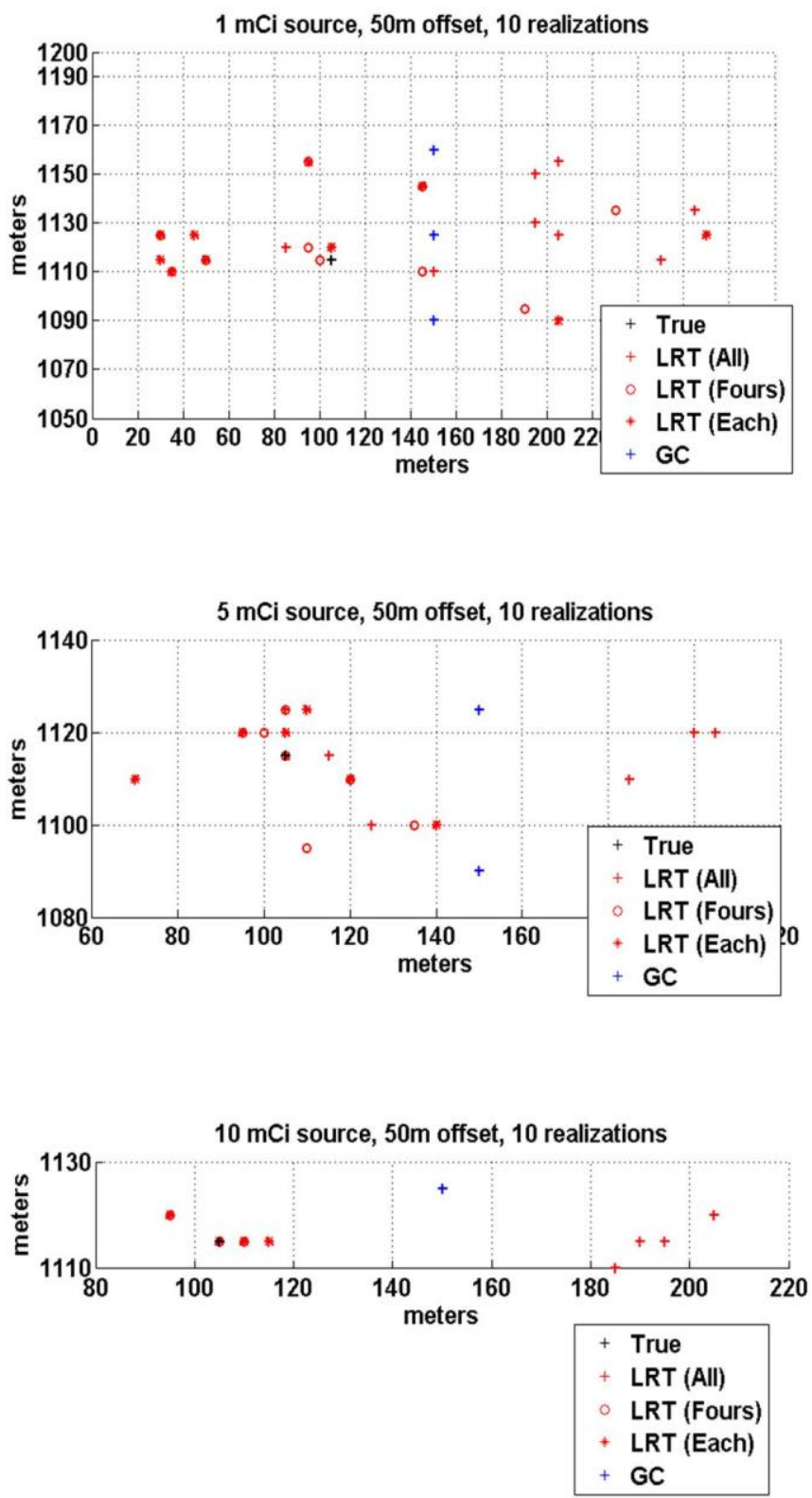

2 Figure 6: Ensemble results for most likely source location at three different source strengths,

3 over 10 realizations and 3 detector binning schemes.

4 Here we can see the distribution of sources, as identified by taking the maximum of the log-LRT.

5 Note that as a detection metric, this is excessively simple since it assumes that a single source is

6 present, but it serves here to illustrate the spread in the distribution of the maximum. For a 1 
$1 \mathrm{mCi}$ source, a wide range of peak locations is found, illustrating the difficulty in localizing based

2 on a very faint signal. Location error along the direction of travel is much lower than lateral to

3 it, reflecting the larger number of measurements along the track. The "All" binning scheme

4 locates the source to either side of the aircraft track, as expected, but the directional binning

5 schemes locate the source on either side as well, due to noise. The location of maximum gross

6 counts varies between three different timesteps/measurement locations.

7 For a $5 \mathrm{mCi}$ source under the same locations, the variation in location is considerably reduced.

8 While the ability to localize lateral to the flight path is still less than along the path, the detector

9 binning schemes can be seen to impact the result significantly: the symmetric "All" binning

10 scheme locates the source on either side of the track, but either of the binning schemes

11 preserving directionality find the source only on the correct side. This greatly reduces the

12 average location error.

13 At $10 \mathrm{mCi}$, the effects seen at $5 \mathrm{mCi}$ are even more pronounced. The spread in location

14 estimates is dropping, although the non-directional binning scheme still finds sources on either 15 side of the track.

16 The average localization error is summarized in Figure 7, as a function of source strength, for

17 three different track offsets with a single flight. The plot summarizes 10 realizations per

18 strength, evaluated at three different binning schemes, for track offset from the true source

19 location by $50 \mathrm{~m}$ and $75 \mathrm{~m}$; and 30 realizations per strength for a track offset by $25 \mathrm{~m}$. 


\section{Location Error}

$2 \quad$ Figure 7: Error in estimated source location over ensemble of single-pass flyovers

3 In most cases, average location error can be seen to decrease as source strength is increased, and

4 location error is lower for smaller offsets. This is in keeping with expected performance as

5 signal is increased and noise is decreased. Interestingly, for the 50 and $75 \mathrm{~m}$ offset flights, the

6 mean error for the "All" binning scheme is larger at $10 \mathrm{mCi}$ than at $5 \mathrm{mCi}$. This may reflect the

7 fact that the "All" scheme finds sources to either side of the track; as noise is reduced the peak

8 location is essentially correct, or off by the offset amount; at higher noise the results are spread

9 out more over the intervening region. Averaging over 10 realizations is not sufficient to fully

10 sample the handoff between a statistically-dominated result to a systematic-dominated result.

11 The "Fours" binning scheme appears to localize equally well to the "Each" binning scheme,

12 indicating that treating the four detector assemblies as a single unit is a valid approach. Finally,

13 note that while having a binning scheme that preserves directionality strongly affects the

14 accuracy of the answer at high source strength, the error at low source strength is nearly 
1 independent of the binning scheme, indicating that the ability of the directional detector to

2 specify source position lateral to the path is lost for very weak sources.

\section{$3 \quad 4.0$ Conclusions}

4 The ARBL method extends conventional maximum likelihood approaches to include real-time

5 updates. The quadratic approximation, limited field of view, and use of a pre-calculated lookup

6 library enable real-time operation for complex detectors and realistic terrain. ARBL is part of a

7 larger effort to improve aerial radiation detection by making use of multi-sensor and auxiliary

8 information. ARBL will make use of improved background estimation techniques as well as

9 spectral anomaly based detection to discriminate sources of interest and reject NORM.

In particular, the results shown in this work assume an accurate real-time estimate of the

11 background, such as might be available from prior aerial survey of the same region. When used

12 with an airborne detection system, this approach will be paired with a background estimation

13 algorithm to provide background maps based on prior measurement, KUT inference or running

14 averages. In addition, this approach will run in tandem with spectroscopic methods which will be

15 used as a corroborating factor in the production of estimated source catalogs (descriptions of

16 candidate sources detected and their locations), allowing for the rejection of naturally occurring

17 radioactive material (NORM) sources.

18 Evaluating the ARBL localization approach with simulated search scenarios, the average error in

19 estimated source location is relatively small, in the range of 10-20 meters for many of the

20 sources considered here. This stands in contrast to the error that would be achieved by taking the

21 location of highest gross count measurement, which is dominated by the field of view. Search

22 performed in this way with a single flyover may have an error of as much as hundreds of meters. 
1 The average error in estimated source location is dominated by errors in the direction normal to

2 the flight path. As illustrated in Figs.4-6, a single-path flyover samples data at many points

3 along the path but only one in the perpendicular direction. As a result, the scatter in estimated

4 source locations is mainly in that direction. In addition, unless information from the individual

5 detectors is used, the left/right asymmetry of the single flyover leads to two areas of estimated

6 source location (one on the left and one on the right), which in turn leads to a high localization

7 error even for stronger sources.

8 The likelihood-based approach is readily generalized to incorporate the complex structure of a

9 directional detector by developing expected responses for individual crystals. Studying the

10 performance of the ARBL algorithm using binning schemes which preserve detector structure

11 ("each" and "fours") or remove it ("all"), we see that the combination of the structured detector

12 and the ARBL algorithm provides a powerful technique for achieving confidence in source

13 location. This technique greatly reduces the error for a single flyover near a source. The added

14 value from this specificity is diminished when multiple "sides" of the source can be viewed, as

15 with the 2-flyover pattern shown in Fig.5. This is as expected, because the additional locations

16 around the source provide the same sort of spatial sensitivity as the structured detectors on a

17 single side. Another way to look at this is that the "left-right symmetry" of a measurement with a

18 single flyover can be broken in either way - by a structured detector, or by measurements at

19 several locations to the left and right of the source. However, since single-pass scenarios are

20 most relevant to rapid urban search, this detector coupled with a likelihood-based approach can

21 allow urban radiological search and localization in a small to reasonable time, whereas a non-

22 structured detector would require a much more exhaustive search pattern to achieve this same

23 spatial confidence. 


\subsection{Acknowledgements}

2 This work has been supported by the US Department of Homeland Security, Domestic Nuclear

3 Detection Office, under competitively awarded contract/IAA HSHQDC-12-X-00376. This

4 support does not constitute an express or implied endorsement on the part of the Government.

5 This report is PNNL-SA-103899. Pacific Northwest National Laboratory is operated for the U.S.

6 Department of Energy by Battelle under Contract DE-AC05-76RL01830.

\section{$7 \quad 6.0$ References}

8 [1] B. L. Dickson, "Recent advances in aerial gamma-ray surveying," Journal of

9 Environmental Radioactivity, vol. 76, pp. 225-236, 20042004.

10

[2] M. J. Kettunen and M. T. Nikkinen, "Fixed-wing gamma measurement for the detection of radioactive materials," Journal of Radioanalytical and Nuclear Chemistry, vol. 263, pp. 241-243, Jan 2005.

[3] J. Towler, B. Krawiec, and K. Kochersberger, "Radiation Mapping in Post-Disaster Environments Using an Autonomous Helicopter," Remote Sensing, vol. 4, pp. 1995-2015, Jul 2012.

[4] Y. Sanada, et al., "Radiation monitoring using an unmanned helicopter in the evacuation zone around the Fukushima Daiichi nuclear power plant," Exploration Geophysics, vol. 45, pp. 3-7, Mar 2014.

[5] D. K. Fagan, S. M. Robinson, and R. C. Runkle, "Statistical methods applied to gammaray spectroscopy algorithms in nuclear security missions," Applied Radiation and Isotopes, vol. 70, pp. 2428-2439, Oct 2012. 
1 [6] D. M. Pfund, R. C. Runkle, K. K. Anderson, and K. D. Jarman, "Examination of countstarved gamma spectra using the method of spectral comparison ratios," Ieee Transactions on Nuclear Science, vol. 54, pp. 1232-1238, Aug 2007.

[7] K. D. Jarman, E. A. Miller, R. S. Wittman, and C. J. Gesh, "BAYESIAN RADIATION SOURCE LOCALIZATION," Nuclear Technology, vol. 175, pp. 326-334, Jul 2011.

[8] E. A. Miller, et al., "Combining Radiography and Passive Measurements for Radiological Threat Detection in Cargo," in 2012 Ieee Nuclear Science Symposium and Medical Imaging Conference Record, B. Yu, Ed., ed, 2012, pp. 657-661.

[9] R. Vilim, R. Klann, and J. Thomas, "INTEGRATED TREATMENT OF DETECTOR ARRAYS FOR SOURCE TRACKING," Nuclear Technology, vol. 175, pp. 314-325, Jul 2011.

[10] S. M. Robinson, S. E. Bender, E. L. Flumerfelt, C. A. LoPresti, and M. L. Woodring, "Time series evaluation of radiation portal monitor data for point source discrimination," Journal of Radioanalytical and Nuclear Chemistry, vol. 282, pp. 883-887, Dec 2009.

[11] D. A. Cooper, et al., "Integration of Inertial Measurement Data for Improved Localization and Tracking of Radiation Sources," 2013 Ieee International Conference on Technologies for Homeland Security, pp. 613-617, 2013. 